\title{
Autism's link to chromatin remodeling, explained
}

\author{
BY GIORGIA GUGLIELMI
}

6 JULY 2021

\section{Listen to this story:}

https://www.spectrumnews.org/wp-content/uploads/2021/07/audiob9cd949d-5ee4-4135-a1fb-5fb96ee6d0f5-encodings.mp3

Autism research has long focused on genes involved in the formation of neurons and the function of synapses. Mutations in these genes were the first to be solidly linked to the condition and its traits. Over the past decade, however, several studies have implicated a second class of genes: those involved in the remodeling of chromatin — the complex of DNA and proteins that makes up chromosomes.

These 'chromatin regulators,' which can influence whether other genes are turned on or off, are sometimes mutated in people with autism or other neurodevelopmental conditions. Scientists are just beginning to understand how these mutations can alter brain development.

\section{Why is chromatin remodeling important?}

If the chromosomes of a single human cell were stretched out and joined together end to end, their DNA would measure about 6.5 feet long. To fit inside a nucleus no wider than one-tenth of a human hair, the DNA strand wraps around histone proteins to form a series of bead-like structures called nucleosomes. Together these beads make up the chromatin.

When a stretch of DNA is tightly packed into a nucleosome, it is inaccessible to the proteins that turn genes on and off by way of a process called transcription. For cells to express the right genes at the right time, their DNA needs to transition from tightly to loosely packed coils, a process carried out by a group of proteins called chromatin remodeling complexes.

Mutations in certain components of these complexes have been tied not only to autism, but also 


\section{Spectrum | Autism Research News}

https://www.spectrumnews.org

to cancer, schizophrenia, intellectual disability and other conditions. The structure of chromatin is also regulated by enzymes that add or remove specific chemical tags on DNA and histone proteins. Mutations in some of these enzymes have been found in people with autism or intellectual disability.

\section{How do we know that chromatin remodeling is linked to autism?}

Genes involved in chromatin regulation are disrupted significantly more often in autistic children than in their unaffected siblings, according to four large sequencing studies published in 2012. Mutations in one such gene, called CHD8, may account for as much as 0.4 percent of autism cases, another study showed.

In fact, the list of genes most confidently linked to autism, gleaned from more recent large-scale sequencing efforts, includes CHD8 and other chromatin regulators, such as ARID1B, ASH1L, KMT2A and SETD5. Even mutations in noncoding portions of the genomes of people with autism appear to affect chromatin structure.

Some autism-linked chromatin regulators, including ACTL6B, constitute part of a chromatin remodeling complex known as BAF. Mutations in ACTL6B are recessive, meaning they are harmful only when a person inherits two copies. Most other mutations of chromatin remodelers implicated in autism are not inherited but arise spontaneously in the egg, sperm or fertilized egg.

\section{How do mutations in chromatin remodeling factors lead to autism?}

It's unclear. Scientists suspect that chromatin regulators are important for brain development. By regulating gene expression, chromatin remodeling may influence the formation and differentiation of neurons. In line with this idea, a large 2019 study revealed a network of autismlinked genes that are involved in chromatin remodeling during the growth of new neurons.

The protein encoded by CHD8, one of the most frequently mutated genes in people with autism, inhibits the function of an important signaling protein called beta-catenin. And mutations in a group of genes that includes CHD8 appear to increase connectivity between brain areas involved in sensory processing. Autistic people with CHD8 mutations often have intellectual disability and a larger-than-average head. Mutations in CHD8 may affect brain growth by controlling the proliferation of cells that give rise to neurons in the cortex - the brain's outer layer.

The BAF complex also has some intriguing connections to brain development. Parts of a neuronspecific chromatin regulator called nBAF are mutated in autism, and researchers have shown that the loss of a BAF component expressed in neuronal progenitors can disrupt the formation of new neurons in mice. Rodents lacking a BAF component expressed in mature neurons have mostly normal neuronal development, but their brain cells do not grow in response to activity as typical neurons do. This leads to autism-like behaviors and deficits in spatial memory and object 
recognition.

\section{What about mutations in enzymes that modify histones?}

Chemical modifications can alter the structure of histone proteins, changing the way genes are exposed and unwound to be read. The genomic patterns of one such modification, known as an acetyl tag, differ between the brains of some autistic people and those of controls.

Indeed, mutations in enzymes that add and remove chemical tags from histones have been linked to autism and other neurodevelopmental conditions. For example, SETD5, a leading autism gene, encodes an enzyme that is thought to add a methyl group to a specific histone, thus altering the expression of hundreds of other genes. Mouse neurons lacking a copy of SETD5 make fewer connections than control neurons do, researchers have found.

\section{Do environmental factors interact with genetic risk factors in altering chromatin structure?}

Scientists know that consuming alcohol or taking drugs such as the epilepsy medication valproate during pregnancy can alter chromatin structure in the fetus. Female mice exposed to the flame retardant polybrominated diphenyl ether (PDBE) before birth had reduced levels of DNA methylation - a chemical alteration to DNA that modifies gene expression - and problems with learning and memory. Some of these problems seem to worsen after exposure to PDBE in a mouse model of Rett syndrome, a rare condition often accompanied by autism. These mice have a mutation in MECP2, a protein that binds to methylated regions of DNA and regulates the expression of other genes. So one possibility is that genes and the environment can combine to modify chromatin structure, resulting in changes to gene expression and behavior.

\section{Could drugs that alter chromatin remodeling help ease some autism traits?}

Maybe. Compounds that influence chromatin remodeling are used as cancer therapies, and some have shown promise for autism, too. For instance, a cancer drug that blocks a family of enzymes called HDACs, which remove acetyl tags from histones, can ease some autism-like features in mice missing part of SHANK3 - a gene mutated in up to 2 percent of people with autism.

Mice lacking SHANK3 have too much of a specific HDAC protein and fewer acetyl groups in their brains than controls do. The mice also show increased expression of two histone-modifying enzymes, EHMT1 and EHMT2, which have been associated with autism and autism-like conditions. Giving the mice a compound that inhibits EHMT enzymes increased the animals' social interest, but it did not have effects on motor function, anxiety or repetitive behaviors.

Because chromatin remodeling happens in all cell types, compounds that block it often have unwanted side effects. Looking for safer alternatives, researchers are exploring other strategies to target chromatin. One promising approach takes advantage of the gene-editing technology 


\section{Spectrum | Autism Research News}

https://www.spectrumnews.org

CRISPR to alter the genome's chemical tags that control chromatin structure and gene expression. This approach could help control the activity of genes linked to autism and ease some of the condition's traits.

Cite this article: https://doi.org/10.53053/PKKO2287 